\title{
Hierarchy and stability of partially synchronous oscillations of diffusively coupled dynamical systems
}

\author{
Vladimir N. Belykh, ${ }^{1}$ Igor V. Belykh, ${ }^{2}$ and Martin Hasler $^{3}$ \\ ${ }^{1}$ Advanced School of General and Applied Physics, Nizhny Novgorod University, 23 Gagarin Avenue, Nizhny Novgorod 603600, Russia \\ ${ }^{2}$ Department of Differential Equations, Institute for Applied Mathematics and Cybernetics, Nizhny Novgorod University, \\ 10 Ul'yanov Street, Nizhny Novgorod 603 005, Russia \\ ${ }^{3}$ Department of Electrical Engineering, Swiss Federal Institute of Technology Lausanne (EPFL), CH-1015 Lausanne, Switzerland
}

(Received 13 July 1999; revised manuscript received 7 April 2000)

\begin{abstract}
The paper presents a qualitative analysis of an array of diffusively coupled identical continuous time dynamical systems. The effects of full, partial, antiphase, and in-phase-antiphase chaotic synchronizations are investigated via the linear invariant manifolds of the corresponding differential equations. The existence of various invariant manifolds, a self-similar behavior, and a hierarchy and embedding of the manifolds of the coupled system are discovered. Sufficient conditions for the stability of the invariant manifolds are obtained via the method of Lyapunov functions. Conditions under which full global synchronization cannot be achieved even for the largest coupling constant are defined. The general rigorous results are illustrated through examples of coupled Lorenz-like and Rössler systems.

PACS number(s): 05.45.- a
\end{abstract}

\section{INTRODUCTION}

Since the pioneering works by Fujisaka and Yamada [1], Afraimovich et al. [2], and Pecora and Carroll [3], synchronization of chaotic systems has attracted a rapidly growing interest in theoretical physics and other fields of science [1-26]. Related to weak attractors and complicated basins of attraction, the concept of chaotic synchronization is now considered one of the basic concepts in the theory of coupled dynamical systems.

The phenomenon of chaotic synchronization has many different applications, e.g., in engineering where it is studied as a tool for transmitting information by using chaotic signals $[3,15,27]$. Numerous studies of the dynamics of coupled chaotic systems have found different types of synchronization phenomena, including the most interesting cases of full and partial synchronization (or clustering [19,21-23]), generalized [12,13], lag [30], and phase synchronization [28,29], riddled basins of attraction [31], attractor bubbling [32], and on-off intermittency [33].

In arrays of coupled identical systems the main types of synchronized regimes are full and partial (cluster) synchronization. In full chaotic synchronization [1-7], all oscillators of the array acquire identical chaotic behaviors even though their initial conditions are different. Partial synchronization [20-24] is observed where only some oscillators synchronize and others do not. Oscillators with identical temporal dynamics form a cluster.

Analytical studies of full and partial synchronization in large ensembles of coupled systems meet some problems, due essentially to the multidimensional phase space of the coupled system. Therefore, phenomena of cluster synchronization in coupled chaotic continuous time systems are usually investigated through numerical analysis [22].

Until recently most studies of cluster synchronization were concerned with coupled map lattices [14,19-21,24] or systems of globally coupled maps [19,23], but the interest has now shifted toward the analysis of coupled continuous time systems, since they have a more direct relation to the properties of real physical systems. The purpose of the present paper is to proceed with a more analytical approach to a description of different types of partial synchronization of identical continuous time oscillators that are coupled in an array with simple scalar diffusive coupling (nearest neighbor interaction). The main problem in this study is finding different embedded invariant linear manifolds corresponding to full, partial, and antiphase synchronization, and obtaining conditions for their stability.

In this paper we consider only identical synchronization dynamical regimes defined by invariant manifolds, and omit cases of generalized, phase, and lag synchronization usually arising in the presence of a parameter mismatch between the oscillators. In addition to full and partial identical synchronization, we study antiphase and in-phase-antiphase synchronization of identical coupled dynamical systems defined by the existence of stable linear transversal invariant manifolds. Such antiphase synchronization is observed in a system of two coupled oscillators where all corresponding variables of the two individual oscillators are equal with opposite sign. In in-phase-antiphase synchronization, one set of the corresponding variables is equal, whereas the other is equal with opposite signs.

In this paper we study the following $K$-dimensional dynamical system, that is composed of diffusively coupled oscillators.

$$
\begin{gathered}
\dot{x}_{i}=P\left(x_{i}, y_{i}\right)+\varepsilon\left(x_{i+1}-2 x_{i}+x_{i-1}\right), \\
\dot{y}_{i}=Q\left(x_{i}, y_{i}\right), \quad i=1,2, \ldots, N,
\end{gathered}
$$

with zero flux $\left(x_{0} \equiv x_{1}, x_{N} \equiv x_{N+1}\right)$ or periodic $\left(x_{0} \equiv x_{N}\right.$, $\left.x_{N+1} \equiv x_{1}\right)$ boundary conditions. In system (1.1), $x_{i} \in R^{1}$ is a scalar variable, and $y_{i} \in R^{m-1}$ a vector. $P: R^{m} \rightarrow R^{1}$ and $Q: R^{m} \rightarrow R^{m-1}$ are continuous and smooth scalar and vector functions, respectively, $\varepsilon>0$ is a coupling parameter, $K$ 
$=N m$ is the dimension of the coupled oscillator system, and $m$ is the dimension of the subsystem.

Denoting the vectors $U_{i}=\left(x_{i}, y_{i}\right)$ and $G\left(U_{i}\right)$ $=\left(P\left(x_{i}, y_{i}\right), Q\left(x_{i}, y_{i}\right)\right)$, we introduce the single subsystem in vector form:

$$
\dot{U}=G(U), \quad U \in R^{m} .
$$

System (1.1) represents an array of nonlinear multidimensional systems of differential equations with the simplest scalar version of diffusive coupling. The phenomena of full and partial synchronization of diffusively coupled oscillators is intimately related to invariant manifolds of system (1.1). We will discover the existence of various embedded linear manifolds related to partial synchronization in in-phase, antiphase, and in-phase-antiphase modes. Depending in an essential way on the number of oscillators $N$ and on the boundary conditions, these manifolds have an ordering that generates a specific hierarchy of synchronous and antiphase oscillations.

We determine the dimension and stability of the partially synchronized states and discuss their order of appearance (stabilization) with increasing coupling. Also, we obtain some conditions under which full global synchronization of diffusively coupled oscillators is impossible even for the largest coupling constant (hereafter we use the term global synchronization for full synchronization arising from all initial conditions). We consider two separate cases of stability conditions: (1) when $\operatorname{det} Q_{y}^{\prime} \neq 0$ for all $x \in R^{1}$ and $y \in R^{m-1}$, and there exists implicit functions $y=q(x)$, and $Q(x, q(x))$ $\equiv 0$; and (2) when $\operatorname{det} Q_{y}^{\prime}$ changes sign at $x=x_{0}$, and the function $y=q(x)$ has a singularity at $x_{0}$. In the first case we determine sufficient conditions for system (1.1) to be globally synchronized and to have globally stable invariant manifolds. In the second case we state that the singularity of the functions $y=q(x)$ leads to the lack of full global synchronization even for the largest coupling parameter $\varepsilon$. Note that this property is related to an active medium which is represented by the single system (1.2) having, for example, in the simplest case $m=2$, a Van der Pol term $Q(x, y)$ $=Q(x, 0)-\alpha\left(x^{2}-1\right) y$ with a characteristic threshold value for $x$.

We illustrate our analytical results through examples of arrays of coupled Lorenz-like systems and of coupled Rössler systems, and finally we discuss the extension of our results to two- and three- dimensional lattices of coupled oscillators and to coupled map lattices with linear and nonlinear symmetrical coupling.

\section{LINEAR INVARIANT MANIFOLDS AND PARTIAL SYNCHRONIZATION}

First we recall the definition of an invariant manifold for a general system of ordinary differential equations (ODE's)

$$
\dot{x}=X(x), x \in R^{N}, X: R^{N} \rightarrow R^{N} .
$$

Consider a vector equation $H(x)=0, H=\left(h_{1}, h_{2}, \ldots, h_{p}\right), p$ $<N$, generating a manifold $M$ of codimension $p, \operatorname{dim} M$ $=N-p$.

Definition 1. Manifold $M$ is an invariant manifold of Eq. (2.1) if

$$
\left.(\operatorname{grad} H \cdot X)\right|_{H=0} \equiv 0,
$$

implying that the vector field (2.1) is tangent to $M$.

In the phase space $R^{K}$ of system (1.1) we consider linear manifolds $M_{N, d}$,

$$
M_{N, d}=\left\{C_{i}^{T} U=0, i=1,2, \ldots, p\right\},
$$

where $\left(c_{i 1}, c_{i 2}, \ldots, c_{i m}\right)^{T}, i=1,2, \ldots, p$ are constant vectors, and $T$ denotes transposition. Obviously, $\operatorname{dim} M_{N, d}$ $=d \cdot m$, where $d=N-p$.

\section{A. Existence of invariant manifolds}

System (1.1) has an invariant manifold $M_{N, 1}=\left\{U_{1}=U_{2}\right.$ $\left.=\cdots=U_{N}\right\}$ which is known as the "diagonal." Full local synchronization takes place if $M_{N, 1}$ is stable in the sense of Lyapunov, and full global synchronization takes place if $M_{N, 1}$ is globally asymptotically stable. Dynamics in $M_{N, 1}$ is generated by the single system (1.2) for each cell of the array. Hence, if the single system has a strange attractor then chaotic synchronization arises.

Below we study the existence of invariant manifolds $M_{N, d}$ with $d>1$, where $d=\operatorname{dim} M_{N, d} / m$ represents the number of constrained variables $x_{j}$ in $M_{N, d}$, or in other words, $d$ is the number of clusters of synchronized elements of an array containing $N$ coupled oscillators.

Definition 2. Let an invariant manifold $M_{N, d}$ be globally asymptotically stable, and let the diagonal $M_{N, 1}$ be unstable. Then the flow in $M_{N, d}$ defines partial synchronization of dimension $\leqslant d$. We also consider the complementary number $p=N-d$ characterizing the penetration of partial synchronization (hereafter, the penetration).

Theorem 1. Let system (1.1) have either zero flux or periodic boundary conditions. Then the following hold.

(1) For odd $N=2 n+1$, system (1.1) has an invariant manifold $\quad M_{N, n+1}=\left\{U_{2 n+1}=U_{1}, U_{2 n}=U_{2}, \ldots, U_{n+3}\right.$ $\left.=U_{n-1}, U_{n+2}=U_{n}\right\}$. Dynamics in $M_{N, n+1}$ is defined by the asymmetric systems

$$
\begin{gathered}
\dot{x}_{1}=P\left(x_{1}, y_{1}\right)+\varepsilon\left(x_{2}-x_{1}\right), \\
\dot{y}_{1}=Q\left(x_{1}, y_{1}\right), \\
\dot{x}_{j}=P\left(x_{j}, y_{j}\right)+\varepsilon\left(x_{j+1}-2 x_{j}+x_{j-1}\right), \\
\dot{y}_{j}=Q\left(x_{j}, y_{j}\right), \quad j=2,3, \ldots, n, \\
\dot{x}_{n+1}=P\left(x_{n+1}, y_{n+1}\right)+2 \varepsilon\left(x_{n}-x_{n+1}\right), \\
\dot{y}_{n+1}=Q\left(x_{n+1}, y_{n+1}\right) .
\end{gathered}
$$

(2) For even $N=2 n$ system (1.1) has an invariant manifold $M_{N, n}=\left\{U_{n+1}=U_{n},=U_{n+2}=U_{n-1}, \ldots, U_{2 n-1}\right.$ $\left.=U_{2}, U_{2 n}=U_{1}\right\}$. Dynamics in $M_{N, n}$ is defined by system (1.1) for $N=n$, with zero-flux boundary conditions: $U_{0}$ $\equiv U_{1}$ and $U_{n+1} \equiv U_{n}$.

Proof. The assertions follow in a straightforward manner from condition (2.2), which is fulfilled both for $M_{N, n+1}$ and $M_{N, n}$ with respect to system (1.1). Equations (2.4) and the equations for the second case are obtained by subtracting the equations of the manifolds in system (1.1). 
We say that the vectors in the manifolds $M_{N, n+1}$ and $M_{N, n}$ have a central ("mirror") symmetry with respect to the middle of the array. In the case of odd $N=(2 n+1)$, the dynamics in $M_{N, n+1}$ defines a spatiotemporal regime under which the elements of the chain are synchronized in pairs relative to the middle $(n+1)$ th element, i.e., the first oscillator is synchronized with the last one, the second oscillator is synchronized with the next to the last oscillator, and so forth. The middle $(n+1)$ th oscillator remains unsynchronized. For the case of even $N=2 n$ all elements are synchronized in pairs symmetrically to the imaginary middle of the chain.

Corollary 1.1. In the case of periodic boundary conditions (BC's) for $N=2 n+1$ (respectively, $N=2 n$ ), each element of the chain may be considered as the first one and, due to theorem 1, the system (1.1) has $2 n(2 n-1)$ other invariant manifolds.

Next, we introduce a particular property of system (1.1) with periodic BC's for even $N=2 n$.

Proposition 1. With periodic BC's and even $N=2 n$, system (1.1) has an invariant manifold $M_{N, 2}=\left\{U_{2 j-1}\right.$ $\left.=U_{1}, U_{2 j}=U_{2}, j=2, \ldots, n\right\}$ with system (1.1) for $N=2$ and the BC's $U_{0}=U_{2}$ and $U_{3}=U_{1}$ in it.

This simple assertion follows from Ref. [14], and was originally stated for one-dimensional coupled maps. Proposition 1 defines the existence of two-cluster synchronization of coupled oscillators in a ring. Note that the two last assertions may be applied to the study of travelling waves via a space shift. Let us consider the existence of other linear invariant manifolds of the general system (1.1) for zero-flux $\mathrm{BC}$ 's and a factorizable number of elements.

Theorem 2. Let the number of elements $N=p \cdot q$, where $p$ and $q$ are arbitrary integers. Then system (1.1) has the invariant manifold $M_{p q, q}=\left\{U_{i}=U_{i+2 q j}, j=1,2, \ldots, \operatorname{Int}[(p\right.$ $-1) / 2]$ and $U_{i}=U_{-i+1+2 q j}, \quad j=1,2, \ldots, \operatorname{Int}(p / 2), \quad i$ $=1,2, \ldots, q\}$, where $\operatorname{Int}(\xi)$ is the integer part of $\xi$.

Proof. Similar to that of theorem 1.

Corollary 2.1. For the same number $N$ written in the reverse order $N=q \cdot p$, we obtain the similar manifold $M_{p q, p}$, and the chain is decomposed into $p$ or $q$ equal subchains and each subchain is identical. Then, within these subspaces, theorem 1 can be applied, and thus smaller subspaces are obtained.

Note that for $N=2 n \cdot q$ the vectors in the manifold $M_{N, q}$ have the central symmetry, and differently for $N$ $=(2 n+1) \cdot q$ the vectors in the manifold $M_{N, q}$ have no central symmetry and are related to an alternating symmetry. Now, as examples, we list the important manifolds for $N$ $=2,3,5$, and 6 in the case of zero-flux BC's.

Example 1. For $N=2$, system (1.1) has a unique invariant manifold $M_{2,1}$ with the single system (1.2) in it.

Example 2. Let $N=3$. Due to theorem 1 there exists the invariant manifold $M_{3,2}=\left\{U_{3}=U_{1}\right\}$ with the following system in it:

$$
\begin{aligned}
& \dot{x}_{1}=P\left(x_{1}, y_{1}\right)+\varepsilon\left(x_{2}-x_{1}\right), \quad \dot{y}_{1}=Q\left(x_{1}, y_{1}\right), \\
& \dot{x}_{2}=P\left(x_{2}, y_{2}\right)+2 \varepsilon\left(x_{1}-x_{2}\right), \quad \dot{y}_{2}=Q\left(x_{2}, y_{2}\right) .
\end{aligned}
$$

Obviously, this asymmetrical system has no other linear in- variant manifolds besides the diagonal $M_{3,1}$. The manifold $M_{3,2}$ defines synchronization between the first and third oscillators.

Example 3. For $N=5$, system (1.1) has the invariant manifold $M_{5,3}=\left\{U_{5}=U_{1}, U_{4}=U_{2}\right\}$, which has the forms

$$
\begin{gathered}
\dot{x}_{1}=P\left(x_{1}, y_{1}\right)+\varepsilon\left(x_{2}-x_{1}\right), \quad \dot{y}_{1}=Q\left(x_{1}, y_{1}\right), \\
\dot{x}_{2}=P\left(x_{2}, y_{2}\right)+\varepsilon\left(x_{3}-2 x_{2}+x_{1}\right), \quad \dot{y}_{2}=Q\left(x_{2}, y_{2}\right), \\
\dot{x}_{3}=P\left(x_{3}, y_{3}\right)+2 \varepsilon\left(x_{2}-x_{3}\right), \quad \dot{y}_{3}=Q\left(x_{3}, y_{3}\right) .
\end{gathered}
$$

It is easy to verify by exhaustive search that system (2.6) has no linear invariant manifolds besides $M_{5,1}$.

Example 4. Let $N=6$. Due to theorem 1 system (1.1) has the invariant manifold $M_{6,3}=\left\{U_{1}=U_{6}, U_{2}=U_{5}, U_{3}=U_{4}\right\}$. Due to theorem 2 there also exist two invariant manifolds $M_{6,2}^{c}=\left\{U_{1}=U_{3}=U_{4}=U_{6}, U_{2}=U_{5}\right\} \quad$ and $\quad M_{6,2}^{a}=\left\{U_{1}=U_{4}\right.$ $\left.=U_{5}, U_{2}=U_{3}=U_{6}\right\}$. Note that the vectors in manifold $M_{6,2}^{c}$ have the central symmetry, and in manifold $M_{6,2}^{a}$ they define the alternating symmetry.

The significant feature of theorems 1 and 2 is the recurrence due to the self-similarity of invariant manifold dynamics as well as the permutation of cofactors of $N$ in theorem 2 . Thus we may generate sequences of embedded manifolds.

\section{B. Embedding of manifolds and hierarchy of dimension of partial synchronization}

First we consider the case where $N$ is a prime number, and zero-flux BC's are applied. Due to theorem 1 system (1.1), in addition to $M_{N, 1}$, has the asymmetrical invariant manifold $M_{2 n+1, n+1}$. Example 3 shows that for $N=5$ there are no other linear manifolds, and exhaustive search shows that system (1.1) for $N=7$ has only two invariant manifolds $M_{7,1}$ and $M_{7,4}$.

We conjecture that for prime (non-factorizable) $N=2 n$ +1 we have only the embedding

$$
M_{2 n+1,1} \subset M_{2 n+1, n+1} \subset R^{K},
$$

and the penetration ordering $0 \rightarrow n \rightarrow 2 n$ has two large gaps. Thus we surmise that in this case there may exist only two spatiotemporal dynamical regimes of identical in-phase synchronization: regimes of full synchronization and of partial synchronization with $(n+1)$ clusters.

For the case of $N$ composed of two prime numbers, $N$ $=(2 n+1)(2 m+1)$, we conjecture by reference to theorems 1 and 2 that instead of the embedding [Eq. (2.7)] we have only an embedding such that the dimension of partial synchronization has a hierarchy

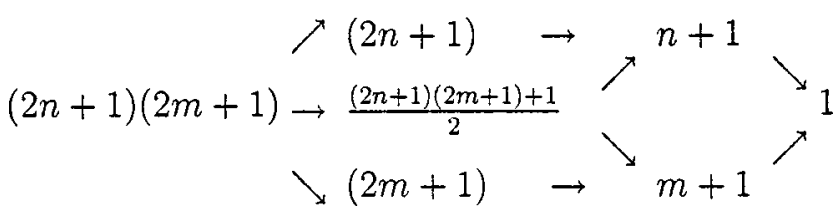

and the penetration ordering has three large gaps.

Consider now the case of even $N=(2 n+1) 2^{k}, n \geqslant 0, k$ $>0$. First we present the existence of invariant manifolds for 
the extreme case of $N=2^{k}(n=0)$. Due to Theorem 1 the system defining the dynamics in $M_{N, n}$ coincides with the initial system (1.1) under the change $N \rightarrow N / 2$. Hence, system (1.1) has complete self-similarity and its invariant manifolds $M_{2^{k}, d_{j}}$, where $d_{j}=2^{j}, j=0,1,2, \ldots, k-1$ are embedded as

$$
M_{2^{k}, 1} \subset M_{2^{k}, 2} \subset M_{2^{k}, 2^{2}} \subset \ldots \subset M_{2^{k}, 2^{k-1}} \subset R^{K},
$$

and for partial synchronization we have the dimension doubling hierarchy

$$
N=2^{k} \rightarrow 2^{k-1} \rightarrow \ldots \rightarrow 2^{2} \rightarrow 2 \rightarrow 1 .
$$

Written in the opposite direction, the penetration $p_{j 1}=2^{k}(1$ $-2^{-j 1}$ ) also has increasing ordering.

In the case $N=(2 n+1) 2^{k}, n>0, k>0$, due to theorems 1 and 2 we obtain an embedding such that the dimension of partial synchronization has the following ordering:

$$
\begin{gathered}
N \rightarrow(2 n+1) 2^{k-1} \rightarrow \ldots \\
\ldots \rightarrow(2 n+1) 2 \nearrow_{\searrow 2 \longrightarrow(n+1) \searrow} \longrightarrow{ }^{\perp} .
\end{gathered}
$$

Here the first path via the prime $(2 n+1)$ corresponds to the central symmetry as determined by theorem 1 , and the second path through ' 2 "' is defined by the second alternating symmetry via theorem 2 . For composed $2 n+1$, embedding (2.11) is continued.

Let $N=r p^{k}$, where $p$ is a prime number and $r$ is an arbitrary integer. Applying theorem 2 recurrently with $N$ taking the value $q_{1} p$, where $q_{1}=r p^{k-1}, q_{2} p, q_{2}=r p^{k-2}, \ldots, q_{j} p$, $q_{j}=r p^{k-j}$, and $q_{k}=r$, we obtain an embedding of the manifolds with alternating symmetry whose dimension of partial synchronization has the following ordering:

$$
r p^{k} \rightarrow r p^{k-1} \rightarrow r p^{k-2} \rightarrow \cdots \rightarrow r .
$$

For $r=1$ this ordering is similar to Eq. (2.10) for any prime number $p=3,5,7, \ldots$. Finally in the general case $N$ $=p_{1}^{k 1} \cdot p_{2}^{k_{2}} \cdots \cdot p_{l}^{k_{l}}$, where $p_{j}$ are prime numbers, theorem 2 is similarly applied when $p$ is taken for any cofactor of $N$.

\section{Transversal manifolds: antiphase and in-phase-antiphase chaotic oscillations}

We define antiphase synchronized chaotic oscillations of two coupled subsystems of system (1.1) with odd symmetry [with the odd functions $P\left(x_{i}, y_{i}\right)$ and $\left.Q\left(x_{i}, y_{i}\right)\right]$ as a chaotic attractor lying in the invariant manifold $\bar{M}_{2,1}=\left\{U_{1}=-U_{2}\right\}$ (here the "bar'" denotes a manifold transversal to $M_{2,1}$ ). We emphasize that the property of system (1.1) with odd $P\left(x_{i}, y_{i}\right)$ and $Q\left(x_{i}, y_{i}\right)$ to be invariant under the involution $(x, y) \rightarrow(-x,-y)$ allows the system to have a manifold transversal to the manifold $M_{N, 1}$. As examples of such systems we can mention, for the case of differential equations, Chua's circuit [26], and for mappings, the standard map [34].
If system (1.1) possesses another type of symmetry (for example, an axial one) then in-phase-antiphase synchronization may be observed. In terms of invariant manifolds chaotic in-phase-antiphase synchronization of two coupled oscillators may be determined as a chaotic attractor lying in the invariant manifold $A=\left\{x_{1}=-x_{2}, y_{1}^{(1)}=-y_{2}^{(1)}, y_{1}^{(2)}\right.$ $=-y_{2}^{(2)}, \ldots, y_{1}^{(i)}=-y_{2}^{(i)}, y_{1}^{(i+1)}=y_{2}^{(i+1)}, \ldots, y_{1}^{(m-1)}$ $\left.=y_{2}^{(m-1)}\right\}$, where $\left(x_{j}, y_{j}^{(1)}, \ldots, y_{j}^{(m-1)}\right), j=1,2$ is the vector of variables of each individual oscillator.

Existence of the manifold $A$ and hence of a dynamical regime is possible only in the case when the functions $P\left(x_{i}, y_{i}\right)$ and $Q\left(x_{i}, y_{i}\right)$ are invariant under the change $\left(x, y^{(1)}, \ldots, y^{(i)}, y^{(i+1)}, \ldots, y^{(m-1)}\right) \rightarrow\left(-x,-y^{(1)}, \ldots\right.$, $\left.-y^{(i)}, y^{(i+1)}, \ldots, y^{(m-1)}\right)$. Chaotic in-phase-antiphase synchronization may take place in the case of coupled Lorenz systems since the Lorenz system has an axial symmetry. In Sec. V, example A, we will give an example of two coupled Lorenz-like systems which demonstrate in-phase-antiphase synchronized oscillations.

Theorem 3 (existence of antiphase synchronized oscillations). Let system (1.1) with zero-flux BC's $U_{0}=U_{1}$, and $U_{N}=U_{N+1}$ have the odd function $G(U)$.

(1) For an even number $N=2 n$ the system has an invariant manifold $\bar{M}_{2 n, n}=\left\{U_{2 n}=-U_{1}, U_{2 n-1}=-U_{2}, \ldots, U_{n+1}\right.$ $\left.=-U_{n}\right\}$ transversal to the manifold $M_{N, n}$, such that the dynamics in it is defined by system $(1.1)$ for $i=1,2, \ldots, n$, with the BC's $U_{0}=U_{1}$ and $U_{n+1}=-U_{n}$.

(2) For an odd number $N=2 n+1$ the system has an invariant manifold $\bar{M}_{2 n+1, n}=\left\{U_{n+1} \equiv 0, U_{2 n+1}=-U_{1}, U_{2 n}\right.$ $\left.=-U_{2}, \ldots, U_{n+2}=-U_{n}\right\}$ transversal to the manifold $M_{N, n+1}$, such that the dynamics in it is defined by system (1.1) for $i=1,2, \ldots, n$, with the BC's $U_{0}=U_{1}$ and $U_{n+1}$ $\equiv 0$.

Proof. Theorem 3 follows from conditions (2.2) valid for Eq. (1.1). The last $n$ equations in Eq. (1.1) for both even and odd numbers $N$ become the first $n$ equations after the change of both variables and sign.

The dynamics in the manifold $\bar{M}_{N, n}$ defines a spatiotemporal regime where each pair of oscillators which are symmetrical with respect to the middle of the chain is antiphase synchronized.

Example 5. Let $N=4$. Then the dynamics of the manifold $\bar{M}_{4,2}=\left\{U_{4}=-U_{1}, U_{3}=-U_{2}\right\}$ is defined by the system

$$
\begin{gathered}
\dot{x}_{1}=P\left(x_{1}, y_{1}\right)+\varepsilon\left(x_{2}-x_{1}\right), \\
\dot{y}_{1}=Q\left(x_{1}, y_{1}\right), \\
\dot{x}_{2}=P\left(x_{2}, y_{2}\right)+\varepsilon\left(x_{1}-3 x_{2}\right), \\
\dot{y}_{2}=Q\left(x_{2}, y_{2}\right) .
\end{gathered}
$$

Example 6. Let $N=5$. Then the dynamics of the manifold $\bar{M}_{5,2}=\left\{U_{3}=0, U_{5}=-U_{1}, U_{4}=-U_{2}\right\}$ is defined by system (2.13) with the term $\left(x_{1}-3 x_{2}\right)$ changed to $\left(x_{1}-2 x_{2}\right)$ in the third equation.

Remark. It is obvious that the transversal manifolds $\bar{M}_{N, d}$ accompany each embedded manifold $M_{N, d}$ when the system in $M_{N, d}$ is similar to Eq. (1.1). 


\section{GLOBAL STABILITY OF INVARIANT MANIFOLDS}

Assuming that $\operatorname{det} Q_{y}^{\prime} \neq 0, x \in R^{1}$, and $y \in R^{m-1}$, we consider the global asymptotic stability of the invariant manifold $M_{N, 1}=\left\{U_{N}=U_{N-1}=\cdots=U_{1}\right\}$, corresponding to full global synchronization of system (1.1). Using the notation

$$
X_{i}=x_{i}-x_{i+1}, \quad Y_{i}=y_{i}-y_{i+1},
$$

we derive the variational equations

$$
\begin{gathered}
\dot{X}_{i}=P_{x}^{\prime}\left(U_{11}\right) \cdot X_{i}+\left(P_{y}^{\prime}\left(U_{12}\right)\right)^{T} \cdot Y_{i}+\varepsilon\left(X_{i+1}-2 X_{i}+X_{i-1}\right), \\
\dot{Y}_{i}=Q_{x}^{\prime}\left(U_{21}\right) \cdot X_{i}+Q_{y}^{\prime}\left(U_{22}\right) \cdot Y_{i} \\
i=1,2, \ldots, N-1
\end{gathered}
$$

with the BC $X_{0}=X_{N}=0$. Here, the derivative $P_{x}^{\prime}$ is a scalar function of $U_{11}, P_{y}^{\prime}$ and $Q_{x}^{\prime}$ are $(m-1)$ column vector functions, $Q_{y}^{\prime}$ is an $(m-1) \times(m-1)$ Jacobi matrix, and $U_{k, l}(k, l=1,2)$ are values coming from the Lagrange meanvalue theorem. These values are time dependent via the solutions $U_{i}(t)$ and $U_{i+1}(t)$ of Eq. (1.1). Note that for infinitesimal $X_{i}$ and $Y_{i}$ system (3.1) becomes a variational system, where $U_{k l}(k, l=1,2)$ are the coordinates of the manifold $M_{N, 1}$, which are driven by the single system.

We introduce the auxiliary system

$$
\begin{gathered}
\dot{X}=-a X+(\alpha(t))^{T} Y, \\
\dot{Y}=c(t) X+B(t) Y,
\end{gathered}
$$

where $a=$ const $>0, \alpha(t)=P_{y}^{\prime}\left(U_{12}(t)\right), \quad c(t)=Q_{x}^{\prime}\left(U_{21}(t)\right)$, and $B(t)=Q_{y}^{\prime}\left(U_{22}(t)\right)$. This system is identical to system (3.1), except that $P_{x}^{\prime}\left(U_{11}\right)$ is changed to $-a$. Denote $b$ $\equiv \max _{U \in R^{m}} P_{x}^{\prime}(U)$, and the value

$$
\nu=\frac{2 \varepsilon-(a+b)}{2 \varepsilon} .
$$

Similar to our results in previous publications $[6,16]$, the next assertion holds.

Theorem 4 (sufficient conditions of full global synchronization). Assume that there exists a positive definite Lyapunov function

$$
V(X, Y)=\left(X^{2}+Y^{T} H Y\right) / 2,
$$

with some symmetrical matrix $H$, such that the derivative with respect to system (3.2) is negative definite for any function $\alpha(t), c(t)$, or $B(t)$ that is generated by solutions of Eq. (3.1). Then for

$$
\begin{array}{r}
0<\nu<1 \quad \text { and } N \leqslant \operatorname{Int}(\pi / \arccos \nu) ; \\
\nu \geqslant 1 \quad \text { and any } N>1
\end{array}
$$

the manifold $M_{N, 1}$ is globally asymptotically stable.

Proof. Consider the function

$$
W=\frac{1}{2} \sum_{i=1}^{N-1}\left(X_{i}^{2}+Y_{i}^{T} H Y_{i}\right)
$$

whose time derivative along trajectories of Eq. (3.1) is negative definite if the quadratic form

$$
\widetilde{Q}=\sum_{i=1}^{N-1}\left(2 \nu X_{i}^{2}-2 X_{i} X_{i+1}\right)
$$

with $X_{N}=0$, is positive definite, which is true under condition (3.5).

Note, that the meaning of the parameter $a(>0)$, which replaces $P_{x}^{\prime}$ that may change sign, is the minimal damping of variable $x$ needed to make system (3.2) globally asymptotically stable.

Corollary 3.1. The sufficient conditions (3.4) and (3.5) make clear physical sense: in order to provide full global synchronization, a large coupling $\varepsilon$ is required, with an increasing number of elements in the array. It is also interesting to note that the larger the damping necessary to stabilize the auxiliary system, the larger the coupling has to be to achieve full global synchronization.

Recall that system (1.1) for $N=(2 n+1) 2^{k}$ has the selfsimilar embedded manifolds $M_{N, 2^{j-1}} \subset M_{N, 2^{j}}, \quad j$ $=1,2, \ldots, k$, with a dynamics in $M_{N, 2^{j}}$ that is of the same form as in Eq. (1.1) but for $N=(2 n+1) 2^{j}$. Hence, due to this self-similarity we obtain the following assertion.

Corollary 2. For $N=(2 n+1) 2^{j}$, conditions (3.4) and (3.5) become conditions of global stability of manifold $M_{N, 1}$ within the manifold $M_{N,(2 n+1) 2^{j}}$.

Observe, that Eq. (3.5) is the only condition of theorem 4 related to the number of oscillators $N$. Then introducing condition (3.5) for the stability of manifold $M_{N, 1}$ along $M_{N,(2 n+1) 2^{j}}$,

$$
(2 n+1) 2^{j} \leqslant \operatorname{Int}(\pi / \arccos \nu)
$$

we obtain an increasing sequence of bifurcation values $\varepsilon_{j}$, $j=1,2, \ldots, k$, corresponding to the increasing dimension of manifolds attracted by $M_{N, 1}$, which thus confirms the conclusion regarding the process of acquiring full global synchronization dimension with increasing parameter $\varepsilon$. One may observe the similar picture for $N=r p^{k}$, where $p$ is a prime number. The next assertion is related to the stability of the invariant manifolds $M_{N, d}, d>1$.

Theorem 5 (sufficient conditions of global stability of $M_{2 n, n}$, and $M_{2 n+1, n+1}$ ). System (3.1) with $N-1$ replaced by $n$, becomes a system of variational equations.

(a) $M_{2 n, n}$, with BC's $X_{0}=X_{1}$ and $X_{n+1}=-X_{n}$. Here $X_{1}=x_{1}-x_{2 n}, X_{2}=x_{2}-x_{2 n-1}, \ldots, X_{n}=x_{n}-x_{n+1}$.

(b) $M_{2 n+1, n+1}$, with BC's $X_{0}=X_{1}$ and $X_{n+1}=0$. In this case $X_{1}=x_{1}-x_{2 n+1}, X_{2}=x_{2}-x_{2 n}, \ldots, X_{n}=x_{n}-x_{n+2}$.

If the conditions of theorem 4 hold for $\nu$ changed to $\nu_{1}$ $=\nu-1 / 2$, and for $N$ changed to $n+1$, then the invariant manifolds $M_{2 n, n}$ and $M_{2 n+1, n+1}$ are globally asymptotically stable.

Proof. Calculation of variational equations for the considered manifolds is straightforward.

The condition that the time derivative of the Lyapunov function (3.6) along trajectories of the system of variational equations must be negative definite is valid when the quadratic form 


$$
\widetilde{Q}_{1}=\sum_{i=1}^{n}\left(2 \nu_{1} X_{i}^{2}-2 X_{i} X_{i+1}\right),
$$

with $X_{0}=X_{n+1}=0$, is positive definite. That is true under conditions (3.5), where $\nu$ and $N$ take the values $\nu_{1}$ and $n$ +1 respectively. Note that the change from $\nu$ to $\nu_{1}$ is the result of a rough approximation of the quadratic form, with the asymmetrical matrix by a symmetrical matrix with $\nu_{1}$ in the main diagonal.

Remark. Similar conditions may formally be derived for the transversal manifolds $\bar{M}_{N, d}$.

We admit that the sufficient conditions of theorems 4 and 5 seem to be far from the real bifurcation values under which full global synchronization arises. Nevertheless, they are useful for a rough estimation of the range of coupling strengths for global synchronization as well as for solving the problem of whether full global synchronization occurs with increasing coupling or not.

We present two alternative routes of transition to full global synchronization, considering, for the sake of simplicity, only prime numbers $N$. Due to our conjecture for this case there exist only two embedded invariant manifolds $M_{2 n+1,1}$ and $M_{2 n+1, n+1}$.

In the first scenario of transition to full global synchronization with increasing coupling from 0 , the manifold $M_{2 n+1, n+1}$ first becomes asymptotically stable but, lying in it, the diagonal $M_{2 n+1,1}$ remains unstable, and partial synchronization with $(n+1)$ clusters arises. With further increased coupling, the diagonal $M_{2 n+1,1}$ becomes stable inside $M_{2 n+1, n+1}$, and hence becomes globally asymptotically stable. All trajectories of system (1.1) lie in the basin of $M_{2 n+1,1}$, and global synchronization of all oscillators of the array arises.

In the second alternative scenario, with increasing coupling the diagonal $M_{2 n+1,1}$ first becomes stable with respect to the trajectories lying in the manifold $M_{2 n+1, n+1}$ while the manifold $M_{2 n+1, n+1}$ is still unstable with respect to the trajectories lying in the phase space outside of it. This implies that under a further increase of the coupling the manifolds $M_{2 n+1, n+1}$ and $M_{2 n+1,1}$ become globally stable simultaneously, and full global synchronization arises right away. Depending on initial conditions it may occur that the system is about to attain $(n+1)$ clusters; however, this regime of "many cluster freedom" decays and gradually develops in time into a single spatially homogeneous cluster defining full synchronization. Note that, within this scenario, the embeddings of invariant manifolds for different numbers $N$, such as, for example, Eq. (2.9), are related to the ordering of the enlargement of the basin of the diagonal $M_{2 n+1,1}$ with increasing coupling.

Now consider the question of the arrangement of equilibria and invariant manifolds of system (1.1) via the problem of global stability. The family of equilibria of system (1.1) is defined by the difference equations

$$
\begin{gathered}
P\left(x_{i}, y_{i}\right)+\varepsilon\left(x_{i+1}-2 x_{i}+x_{i-1}\right)=0, \\
Q\left(x_{i}, y_{i}\right)=0, \quad i=1,2, \ldots, N
\end{gathered}
$$

which for a finite number $N$ has a finite number $l_{c}$ of solutions that is usually less than $l_{s}^{N}$, where $l_{s}$ is the number of equilibria of single system (1.2). Equation (3.8) is associated with the "spatial'" two-dimensional $[34,14]\left(x_{i}, z_{i} \equiv x_{i-1}\right)$ $\rightarrow\left(x_{i+1}, z_{i+1}\right)$, whose family of trajectories

$$
\begin{aligned}
F & =\left\{\left(x_{1}, x_{1}\right) \rightarrow\left(x_{2}, x_{1}\right) \rightarrow \cdots\right. \\
& \left.\rightarrow\left(x_{N}, x_{N-1}\right) \rightarrow\left(x_{N}, x_{N}\right)\right\}
\end{aligned}
$$

is the set of equilibria of system (1.1), and satisfies zero-flux boundary conditions.

For a fairly small $\varepsilon$ a subset $F_{M}$ of the family $F$ lies in the embedded manifolds, and its massive complement $F \backslash F_{M}$ lies in the phase space $M_{N, N}$ outside of the manifolds. With increasing coupling strength the invariant manifold of the largest dimension $M_{N, d_{1}}$ becomes globally stable, and the complementary part of equilibria $F \backslash F_{M}$ disappears via bifurcations of Eq. (3.9).

Further increase of $\varepsilon$ (up to the values for global stability of the next low dimensional manifold $M_{N, d_{2}}$ ) yields the disappearance of the part of equilibria settled in $M_{N, d_{1}}$ which is the complement to equilibria in $M_{N, d_{2}}$, and so forth. When full global synchronization occurs, all $l_{s}^{N}-l_{s}$ equilibria lying outside of the diagonal $M_{N, 1}$ have disappeared via bifurcations of map (3.9). In this connection the following problem arises: what is the relation between the bifurcations in which the equilibria of Eq. (1.1) disappear and the bifurcations of the onset of global stability of the cluster synchronization manifolds? Section IV demonstrates additional aspects of the complexity of this problem.

\section{IMPOSSIBILITY OF FULL GLOBAL SYNCHRONIZATION}

For a large number of examples of diffusively coupled systems of differential equations, full global synchronization arises with increasing coupling, and remains up to infinite coupling strength. This typical transition occurs through a sequence of bifurcations corresponding either to a decrease of the dimension of the partial synchronization or to the enlargement of the basin of attraction for the diagonal manifold $M_{2 n+1,1}$, i.e., via the above mentioned alternative scenarios. In this section we obtain some criteria for peculiarities of an individual array oscillator, and for the place of diffusive scalar coupling in Eq. (1.1), such that the latter bifurcational scenario is broken and full global synchronization cannot be achieved even for the largest coupling strength.

Note that a similar phenomenon was observed for a chain of coupled Rössler systems in which the stability of the synchronization regime was lost with an increase of coupling $[7,10]$. These desynchronization bifurcations were called short-wavelength bifurcations [7].

The reason for the absence of full global synchronization in our criteria is the existence of equilibria outside the diagonal $M_{N, 1}$ which remain for any large coupling due to peculiarities of system (1.1). In this case the behavior of system (1.1) depends essentially on whether these equilibria are a unique limiting set or if they have some neighboring attractor outside the diagonal. Assume that the system (1.1) has the following properties. 
(1) Equation $Q(x, y)=0$ has a real solution, with respect to $y$, in the form of an explicit function $y=q(x): R^{1}$ $\rightarrow R^{m-1}$ in some interval $I_{0}=\left\{|x|<\delta_{0}\right\}$, such that $q(x)$ has a singularity at $x=0$ because det $Q_{y}^{\prime}(x, y)$ changes sign at $x$ $=0$.

(2) The function

$$
f(x) \triangleq-P(x, q(x))
$$

has a singularity at $x=0$, and satisfies the conditions

$$
\begin{gathered}
\lim _{x \rightarrow \pm 0} f(x)=\mp \infty \\
f(x)>0 \text { for }-\delta_{0}<x 0, f(x)<0 \text { for } 0<x<\delta_{0} .
\end{gathered}
$$

Note that any singularity may be shifted to zero without loss of generality.

\section{A. Two coupled oscillators}

First consider two coupled oscillators of form (1.1) under conditions (4.2):

$$
\begin{gathered}
\dot{x}_{i}=P\left(x_{i}, y_{i}\right)+\varepsilon\left(X_{i+1}-x_{i}\right), \\
\dot{y}_{i}=Q\left(x_{i}, y_{i}\right), \quad i=1,2, \quad x_{3}=x_{1} .
\end{gathered}
$$

This system has an invariant manifold $M_{2,1}=\left\{x_{2}=x_{1}, y_{2}\right.$ $\left.=y_{1}\right\}$, whose global asymptotic stability is equivalent to the global synchronization of the two systems. Equilibria of Eqs. (4.3) are the solutions of

$$
\begin{gathered}
x_{2}=x_{1}+\mu f\left(x_{1}\right) \triangleq \varphi\left(x_{1}\right), \\
x_{1}=x_{2}+\mu f\left(x_{2}\right)=\varphi^{-1}\left(x_{2}\right),
\end{gathered}
$$

where $\mu=\varepsilon^{-1}>0$, and $\varphi^{-1}$ is the inverse function of $\varphi$.

Lemma 1. If system (4.3) satisfies condition (4.2) then there exists a value $\varepsilon_{0}>0$ such that for $\varepsilon>\varepsilon_{0}$ the individual systems of Eq. (4.3) cannot be globally synchronized.

Proof. Due to Eq. (4.2) the function $x_{2}=\varphi\left(x_{1}\right)$ for small $\mu$ is close to $x_{2}=x_{1}$ for $x_{1} \notin\left(I_{0}, I_{1}\right)$, where $I_{1}$ $=\left\{|x|<\delta_{1}(\mu)\right\}, 0<\delta_{1}(\mu)<\delta_{0}$, and $\lim _{\mu \rightarrow 0} \delta_{1}(\mu)=0$, and $\varphi\left(x_{1}\right)$ satisfies Eq. (4.2) for $x_{1} \in I_{1}$.

Hence the function $\varphi\left(x_{1}\right)$ has two branches: $\varphi_{1}(x)>x_{1}$ for $x_{1}<0$ and $\varphi_{2}\left(x_{1}\right)<x_{1}$ for $x_{1}>0$. On the other hand, the function $x_{1}=\varphi^{-1}\left(x_{2}\right)$ in Eq. (4.4) as the inverse function for $\varphi\left(x_{1}\right)$ has the same properties as $\varphi\left(x_{2}\right)$, symmetrical with respect to the diagonal $\left\{x_{2}=x_{1}\right\}$. Thus there exists a $\mu_{0}>0$ such that for $\mu<\mu_{0}\left(\varepsilon>\varepsilon_{0}\right)$ the branches $\varphi_{1}\left(x_{1}\right)$ and $\varphi_{2}^{-1}\left(x_{2}\right)\left[\varphi_{2}\left(x_{1}\right)\right.$ and $\left.\varphi_{1}^{-1}\left(x_{2}\right)\right]$ have an intersection at a point $E_{1}\left(x_{1}^{(1)}, x_{2}^{(1)}\right) \notin\left\{x_{1}=x_{2}\right\} \quad$ [in the symmetrical point $E_{2}\left(-x_{1}^{(1)},-x_{2}^{(1)}\right)$, respectively]. Hence, as $E_{1,2}$ are equilibria of Eqs. (4.3) outside $M_{2,1}$ (see Fig. 1) so for $\varepsilon>\varepsilon_{0}$ the invariant manifold $M_{2,1}$ of system (4.3) cannot be globally asymptotically stable.

\section{B. Three coupled oscillators}

Now consider three coupled oscillators [Eq. (1.1)]. This system has an invariant manifold $M_{3,2}=\left\{x_{3}=x_{1}, y_{3}=y_{1}\right\}$ on which the dynamics is given by

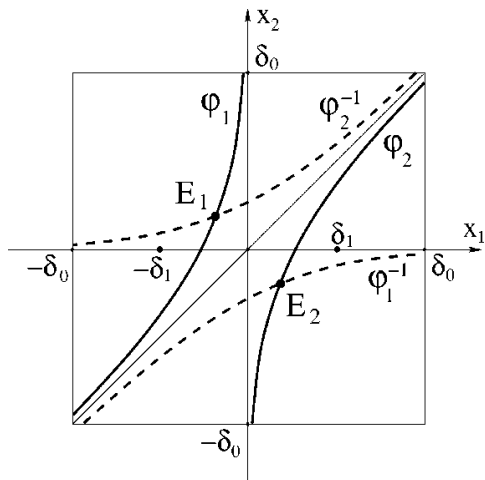

FIG. 1. The functions $\varphi_{1}\left(x_{1}\right)$ and $\varphi_{2}\left(x_{1}\right)$ and the inverse functions $\varphi_{1}^{-1}\left(x_{2}\right)$ and $\varphi_{2}^{-1}\left(x_{2}\right)$. The two points of their intersection give the $x$ coordinates of the equilibrium points $E_{1}$ and $E_{2}$ of system (1.1)

$$
\begin{array}{cc}
\dot{x}_{1}=P\left(x_{1}, y_{1}\right)+\varepsilon\left(x_{2}-x_{1}\right), & \dot{y}_{1}=Q\left(x_{1}, y_{1}\right), \\
\dot{x}_{2}=P\left(x_{2}, y_{2}\right)+2 \varepsilon\left(x_{1}-x_{2}\right), & \dot{y}_{2}=Q\left(x_{2}, y_{2}\right) .
\end{array}
$$

This system again has a submanifold $M_{3,1}=\left\{x_{1}=x_{2}\right.$ $\left.=x_{3}, y_{1}=y_{2}=y_{3}\right\}, M_{3,1} \subset M_{3,2}$, such that if both $M_{3,2}$ and $M_{3,1}$ are globally asymptotically stable then system (1.1) for $N=3$ is globally synchronized.

We consider the relative stability of $M_{3,1}$ with respect to the trajectories of (4.5) in the manifold $M_{3,2}$. Equilibria of Eqs. (4.5) are given by the system

$$
x_{2}=x_{1}+\mu f\left(x_{1}\right) \equiv \varphi\left(x_{1}\right), \quad x_{1}=x_{2}+\mu f\left(x_{2}\right) \equiv \varphi\left(x_{2}\right),
$$

where $\mu=\varepsilon^{-1}>0$ and $f$ is defined by Eq. (4.1).

Lemma 2. Let conditions (4.2) hold. Then there exists a value $\varepsilon_{0}>0$ such that for $\varepsilon>\varepsilon_{0}$ the invariant manifold $M_{3,1}$ cannot be absolutely stable, and system (1.1) for $N=3$ cannot be globally synchronized.

Proof. The functions in Eqs. (4.6) are not the inverse of each other. Nevertheless we prove the existence of two asymmetric equilibrium points $\widetilde{E}_{1,2} \notin M_{3,1}$ in a straightforward manner as for lemma 1 (see Fig. 1), and thus obtain assertion of the lemma.

Remark 1 . In contrast to $M_{3,1}$, the $(2 \times m)$-dimensional invariant manifold $M_{3,2}$ can be globally asymptotically stable because it contains the diagonal $M_{3,1}$ and the two equilibria $E_{1,2}$, and, hence, partial synchronization is possible in the case when det $Q_{y}^{\prime}(0, y)=0$. In the following we demonstrate this through examples of arrays of coupled Lorenz-like systems and of coupled Rössler systems.

\section{C. $N$ coupled oscillators}

Theorem 6. Let conditions (4.2) hold. Then all oscillators of the array (1.1), for the numbers $N$ divisible by 2 or by 3 , cannot be globally synchronized.

Proof. Recall that for $N=N^{\prime} \cdot 2$ and $N=N^{\prime \prime} \cdot 3$, system (1.1) has an invariant manifold $M_{N, 2}$ which contains the manifold $M_{N, 1}$. In accordance with lemmas 1 and 2 , the diagonal $M_{N, 1}$ is not globally asymptotically stable in $M_{N, 2}$, and therefore all individual subsystems of system (1.1) can not be globally synchronized. 
Remark 2. The conclusions of theorem 6 allow the diagonal $M_{N, 1}$ to be locally stable, and full local synchronization may arise (see Sec. VI, example B).

Finally we note that the role of the equilibria $E_{1,2}$ in the process of desynchronization depends on the behavior of its unstable manifolds $W^{u}\left(E_{1,2}\right)$. That is, if $W^{u}\left(E_{1,2}\right)$ are entirely attracted by the diagonal $M_{N, 1}$ then the existence of equilibria $E_{1,2}$ may be considered as unessential for the process of synchronization. A completely different situation arises when $W^{u}\left(E_{1,2}\right)$ are attracted by both the diagonal and by some other attractor, say, by an attracting orbit enclosing $M_{N, 1}$. In this case the existence of these equilibria has direct relation to the mechanism of desynchronization.

\section{EXAMPLE A}

Let us illustrate the main theorems and assumptions by examples.

\section{A. Two coupled Lorenz-like systems}

We consider the following two coupled systems of differential equations:

$$
\begin{gathered}
\dot{x}_{1}=-\sigma\left(x_{1}-y_{1}\right)+\varepsilon\left(x_{2}-x_{1}\right), \\
\dot{y}_{1}=\rho x_{1}-\left(c x_{1}^{2}-h\right) y_{1}-x_{1} z_{1}, \\
\dot{z}_{1}=-b z_{1}+x_{1} y_{1}, \\
\dot{x}_{2}=-\sigma\left(x_{2}-y_{2}\right)+\varepsilon\left(x_{1}-x_{2}\right), \\
\dot{y}_{2}=\rho x_{2}-\left(c x_{2}^{2}-h\right) y_{2}-x_{2} z_{2}, \\
\dot{z}_{2}=-b z_{2}+x_{2} y_{2} .
\end{gathered}
$$

System (5.1) is an example of two diffusively coupled Lorenz-like systems but with a Van der Pol-like term. Actually, for $c=0$ and $h=-1$ we have two coupled conventional Lorenz systems.

As follows from theorem 5 and, for example, from Refs. $[2,16]$, the coupled original Lorenz systems exhibit full global chaotic synchronization with increasing coupling. The invariant manifold $M_{2,1}:\left\{x_{1}=x_{2}, y_{1}=y_{2}, z_{1}=z_{2}\right\}$ becomes globally asymptotically stable, and attracts all trajectories of the system for $t \rightarrow \infty$.

Now let us study the behavior of the coupled modified Lorenz system (5.1). Coordinates of the equilibria of the system (5.1) are defined by the system of equations

$$
\begin{aligned}
& \varepsilon\left(x_{2}-x_{1}\right)=\sigma\left(x_{1}-\frac{\rho x_{1}}{(c+1 / b) x_{1}^{2}-h}\right), \\
& \varepsilon\left(x_{1}-x_{2}\right)=\sigma\left(x_{2}-\frac{\rho x_{2}}{(c+1 / b) x_{2}^{2}-h}\right),
\end{aligned}
$$

which can be rewritten as

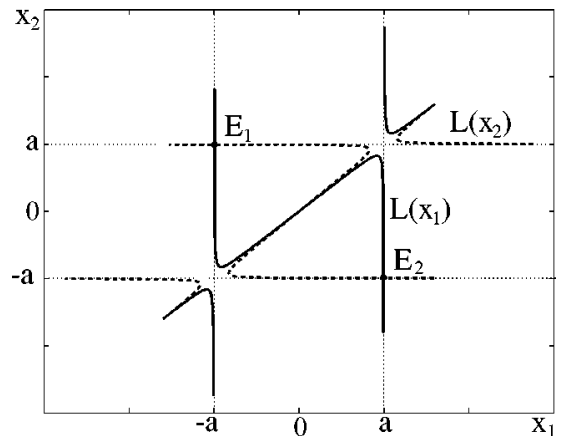

FIG. 2. The curves $x_{2}=L\left(x_{1}\right)$ (solid line) and $x_{1}=L\left(x_{2}\right)$ (dashed line), like the curves of Fig. 1 but for the concrete system (5.1). The intersections give the $x$ coordinates of the equilibria for system (5.1). Equilibria $E_{1}$ and $E_{2}$ exist for any coupling, and lie outside the diagonal $x_{1}=x_{2}$.

$$
\begin{aligned}
& x_{2}=x_{1}+\frac{\sigma}{\varepsilon}\left(x_{1}-\frac{\rho x_{1}}{(c+1 / b) x_{1}^{2}-h}\right)=L\left(x_{1}\right), \\
& x_{1}=x_{2}+\frac{\sigma}{\varepsilon}\left(x_{2}-\frac{\rho x_{2}}{(c+1 / b) x_{2}^{2}-h}\right)=L\left(x_{2}\right) .
\end{aligned}
$$

The solutions of Eqs. (5.3) are the period-2 cycles of the following mapping:

$$
\bar{x}=L(x)=x+\frac{\sigma}{\varepsilon}\left(x-\frac{\rho x}{(c+1 / b) x^{2}-h}\right) .
$$

These solutions are defined by the points of intersection of the curves $x_{2}=L\left(x_{1}\right)$ and $x_{1}=L\left(x_{2}\right)$ on the plane $\left(x_{1}, x_{2}\right)$. It is obvious that they are symmetrical with respect to the diagonal $x_{1}=x_{2}$.

In contrast to the case of coupled original Lorenz systems, the second terms in Eqs. (5.3) do not vanish even with infinite coupling due to a singularity of the function $L$ in the points $x_{1,2}= \pm a$, where $a=\sqrt{h /(c+1 / b)}$. According to lemma 1 , and due to the existence of the two equilibrium points, there is no global synchronization in the system.

In Fig. 2 the curves $x_{2}=L\left(x_{1}\right)$ and $x_{1}=L\left(x_{2}\right)$ are shown. They have intersection points not only on the diagonal but also outside of it. The equilibria $E_{1}$ and $E_{2}$ are preserved for any value of the coupling parameter. They are saddle points.

In Fig. 3 it is shown that there is no global synchronization regime with increasing coupling parameter $\varepsilon$. Figure 3(a) presents a one-dimensional bifurcation diagram for the difference $x_{1}-x_{2}$ on the changing coupling $\varepsilon$. The difference between the corresponding variables $\left(x_{1}\right.$ and $\left.x_{2}\right)$ of the oscillators is plotted vertically (500 points) for each fixed $\varepsilon$ and for fixed initial conditions $\left(x_{1} \neq x_{2}\right)$ at each step of $\varepsilon$ (the number of preiterates equals 20000 points). The nonsynchronized regime $\left(x_{1} \neq x_{2}\right)$ is preserved up to infinite $\varepsilon$. Figure 3(b) shows a projection of a typical non-synchronized chaotic trajectory on the plane $\left(x_{1}, x_{2}\right)$ for a large $\varepsilon$.

System (5.1) has an axial symmetry, as does the original Lorenz system. This means that system (5.1) is invariant under the involution $(x, y, z) \rightarrow(-x,-y, z)$. This fact allows the system to have in-phase-antiphase synchronized oscillations. Being in good accordance with the theory, numerical 


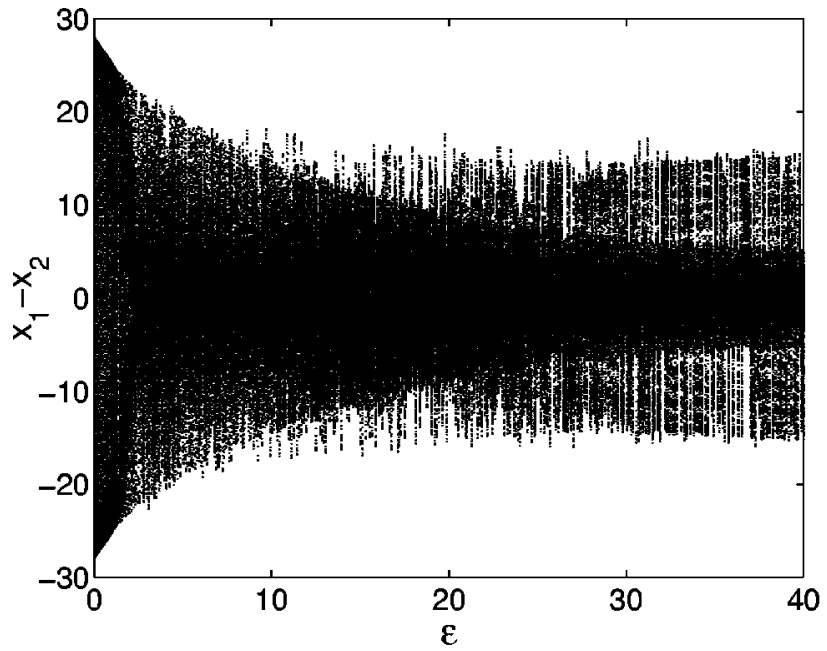

(a)

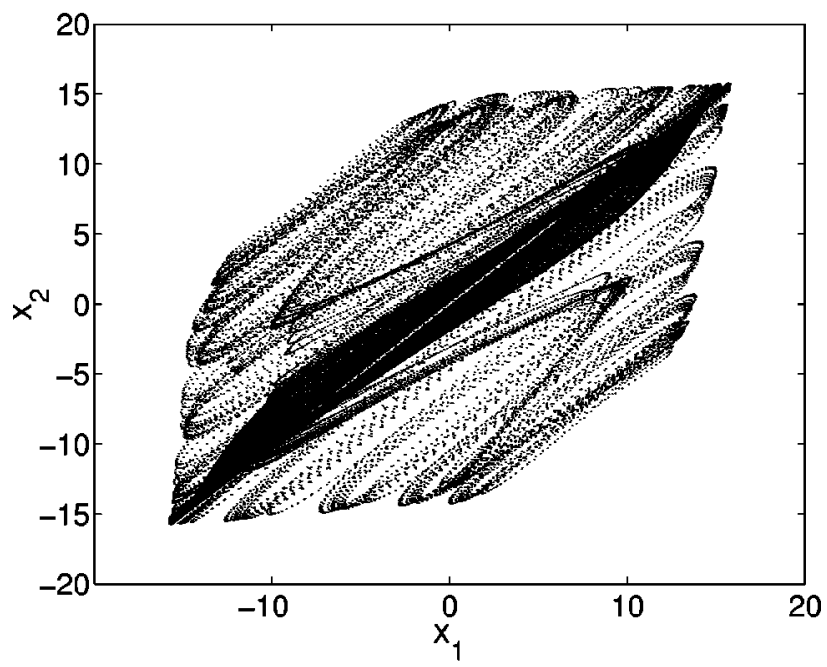

(b)

FIG. 3. Two Lorenz-like systems. (a) Bifurcation diagram for the dependence of the difference $x_{1}-x_{2}$ on the coupling $\varepsilon$. The difference between $x_{1}$ and $x_{2}$ of the subsystems is plotted vertically (500 points) for each fixed $\varepsilon$ for fixed initial conditions $\left(x_{1} \neq x_{2}\right)$ at each step of $\varepsilon$ (20000 preiterates). The other parameters are $\sigma$ $=10, \rho=28, c=0.2, h=20$, and $b=1.6$. (b) Projection of the chaotic attractor on the plane $\left(x_{1}, x_{2}\right)$ for $\varepsilon=110$.

analysis shows that for some interval of $\varepsilon$ (from small $\varepsilon$ up to $\varepsilon \approx 1.25$ ), in-phase-antiphase synchronization is indeed observed in the system.

Corresponding to these solutions, the manifold $A=\left\{x_{1}\right.$ $\left.=-x_{2}, y_{1}=-y_{2}, z_{1}=z_{2}\right\}$ exists, and attracts all trajectories except the stable manifolds of the saddles $E_{1}$ and $E_{2}$. After $\varepsilon \approx 1.25$ this in-phase-antiphase mode loses its stability, and only chaotic behavior exists. Figure 4 illustrates this process. A bifurcation diagram for the dependence of $x_{1}+x_{2}$ on $\varepsilon$ is presented in Fig. 4(a). The line $\left(x_{1}+x_{2}=0\right)$ corresponds to antiphase synchronized $x_{1}$ and $x_{2}$ for $\varepsilon \leqslant 1.25$. A bifurcation diagram $\left[\left(z_{1}-z_{2}\right), \varepsilon\right]$ is shown in Fig. 4(b). The coordinates $z_{1}$ and $z_{2}$ are in-phase synchronized up to the same value of $\varepsilon$. Thus the subsystems of Eqs. (5.1) are in-phase-antiphase synchronized.

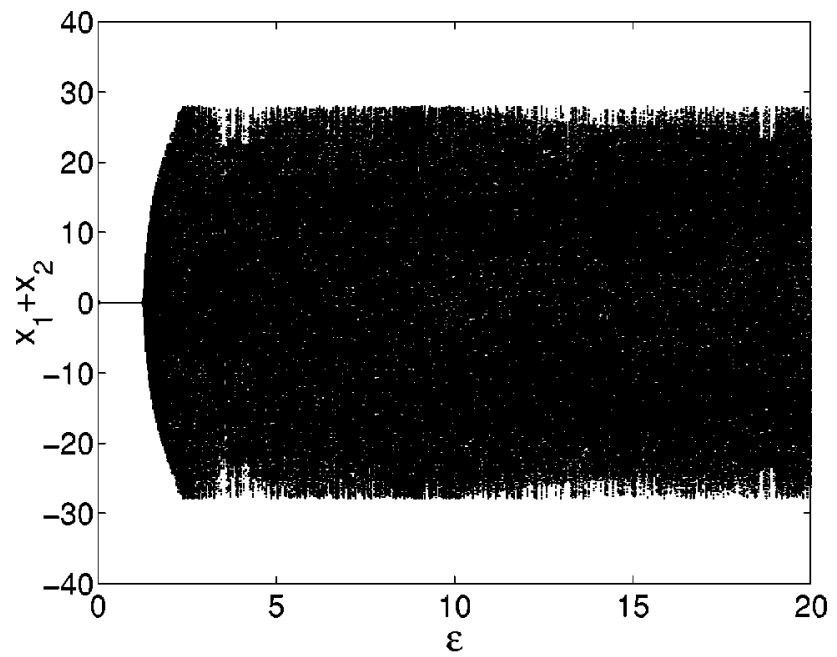

(a)

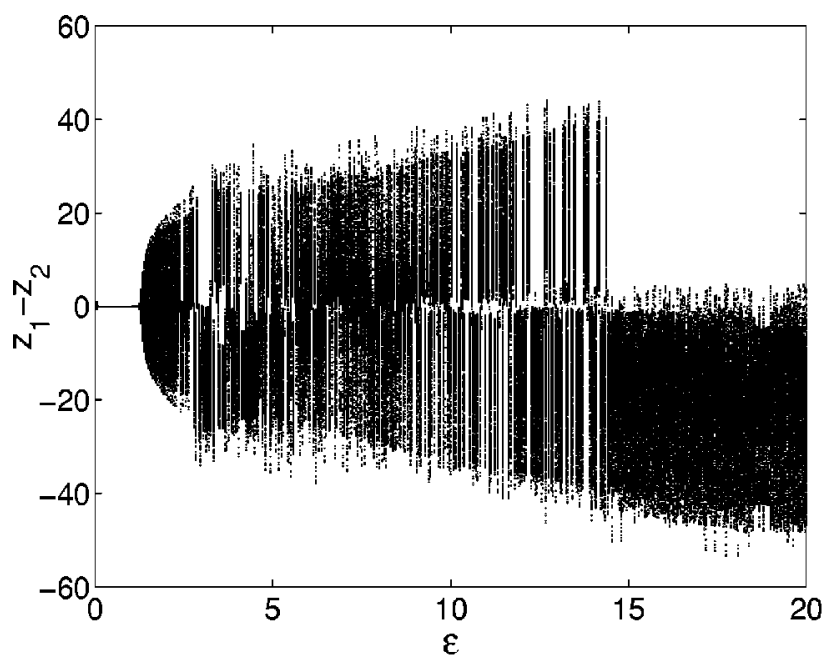

(b)

FIG. 4. In-phase-antiphase synchronization. (a) Bifurcation diagram for the dependence of $x_{1}+x_{2}$ on $\varepsilon$. The line $\left(x_{1}+x_{2}=0\right)$ corresponds to antiphase synchronized $x_{1}$ and $x_{2}$ for $\varepsilon \leqslant 1.25$. (b) Bifurcation diagram $\left[\left(z_{1}-z_{2}\right), \varepsilon\right]$. The coordinates $z_{1}$ and $z_{2}$ are in-phase synchronized up to $\varepsilon=1.25$.

In this paper we do not discuss details of how the impossibility of full global synchronization depends on the place and type of coupling. We only note that the displacement of the same coupling term of system (5.1) to the second equations of the subsystems eliminates the singularity in the function (4.1), and full global synchronization arises.

\section{B. Three coupled Lorenz-like systems}

Now we consider three diffusively coupled oscillators

$$
\begin{gathered}
\dot{x}_{i}=-\sigma\left(x_{i}-y_{i}\right)+\varepsilon\left(x_{i+1}-2 x_{i}+x_{i-1}\right), \\
\dot{y}_{i}=\rho x_{i}-\left(c x_{i}^{2}-h\right) y_{i}-x_{i} z_{i}, \\
\dot{z}_{i}=-b z_{i}+x_{i} y_{i}, \quad i=1,2,3,
\end{gathered}
$$

with zero-flux BC's $x_{0}=x_{1}$ and $x_{4}=x_{3}$. 


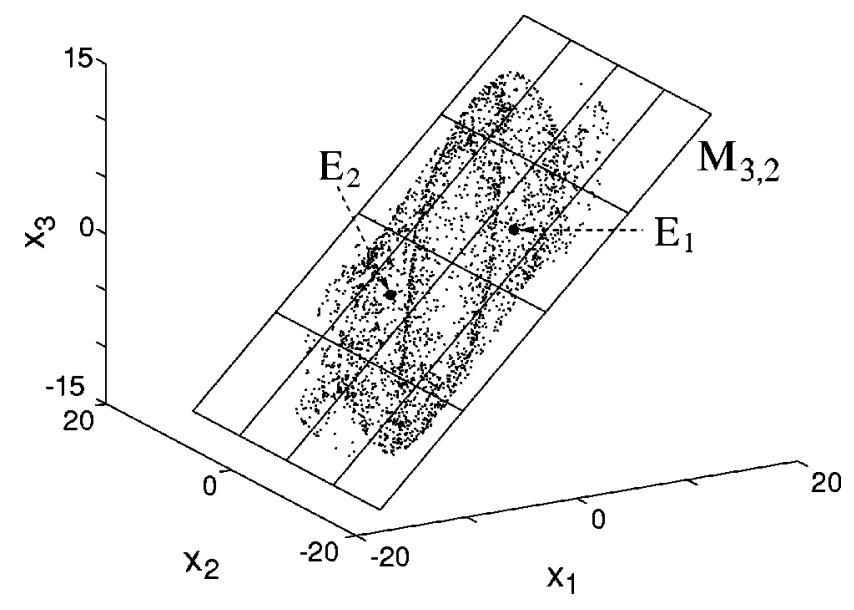

FIG. 5. Globally stable manifold $M_{3,2}$ in the space $\left(x_{1}, x_{2}, x_{3}\right)$. We show the chaotic trajectory of Eq. (5.4), and the two saddle foci $E_{1}$ and $E_{2}$ that lie in it. $\varepsilon=30$, and the other parameters are the same as in Fig. 3.

According to theorem 6, the individual subsystems of Eqs. (5.4) cannot be globally synchronized (the invariant manifold $M_{3,1}$ is not globally stable because of the existence of the equilibria outside the diagonal). The unstable diagonal $M_{3,1}$ with the equilibria $E_{1}$ and $E_{2}$ lies in the manifold $M_{3,2}=\left\{x_{1}=x_{3}, y_{1}=y_{3}, z_{1}=z_{3}\right\}$ which can be stable. In our case $M_{3,2}$ is indeed stable, so that partial synchronization takes place.

A projection of the stable manifold $M_{3,2}$ onto the space $\left(x_{1}, x_{2}, x_{3}\right)$ is shown in Fig. 5. $M_{3,2}$ attracts all trajectories of system (5.4). Corresponding to the absence of full global synchronization, a chaotic attractor lies in $M_{3,2}$ together with two saddle foci $E_{1}$ and $E_{2}$.

Figure 6(a) shows a one-dimensional bifurcation diagram for $x_{1}-x_{2}$ with respect to changing $\varepsilon$. There is no synchronization between the variables of the first and the second oscillators of Eqs. (5.4) for any $\varepsilon$.

It is illustrated in Fig. 6(b) that partial synchronization occurs in system (5.4). For $\varepsilon \approx 19$ the manifold $M_{3,2}$ becomes stable, and the first and third oscillators start to became globally synchronized.

We emphasize that for the two and three coupled Lorenzlike systems, unstable manifolds of the equilibria $E_{1,2}$ are attracted by a complicated attractor lying outside the diagonal. Hence in such cases the existence of these equilibria with this arrangement of their unstable manifolds may serve as a criterion of desynchronization.

\section{EXAMPLE B}

\section{A. Two coupled Rössler systems}

We now consider two diffusively coupled Rössler systems

$$
\begin{gathered}
\dot{x}_{1}=-\left(y_{1}+z_{1}\right)+\varepsilon\left(x_{2}-x_{1}\right), \\
\dot{y}_{1}=x_{1}+a y_{1}, \\
\dot{z}_{1}=b+\left(x_{1}-c\right) z_{1} ; \\
\dot{x}_{2}=-\left(y_{2}+z_{2}\right)+\varepsilon\left(x_{1}-x_{2}\right),
\end{gathered}
$$

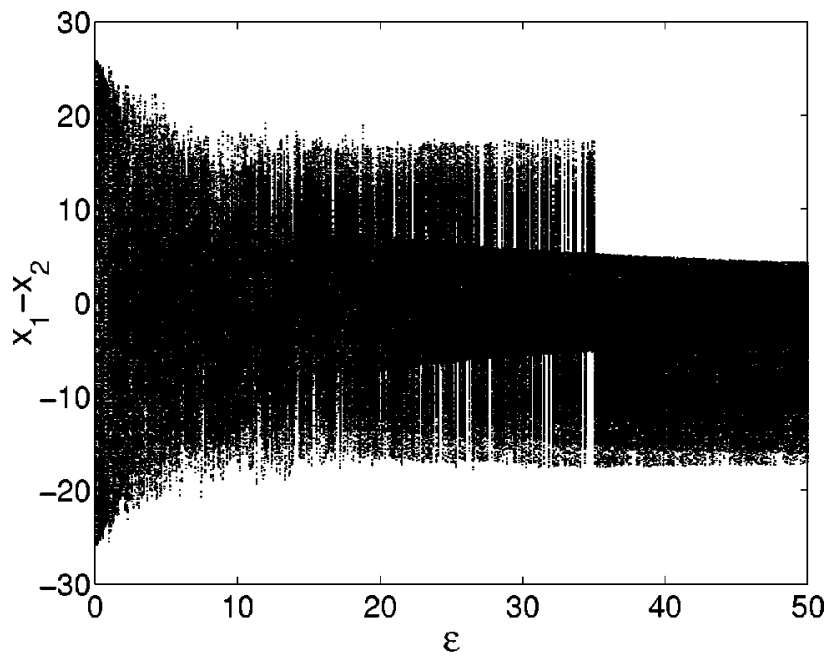

(a)

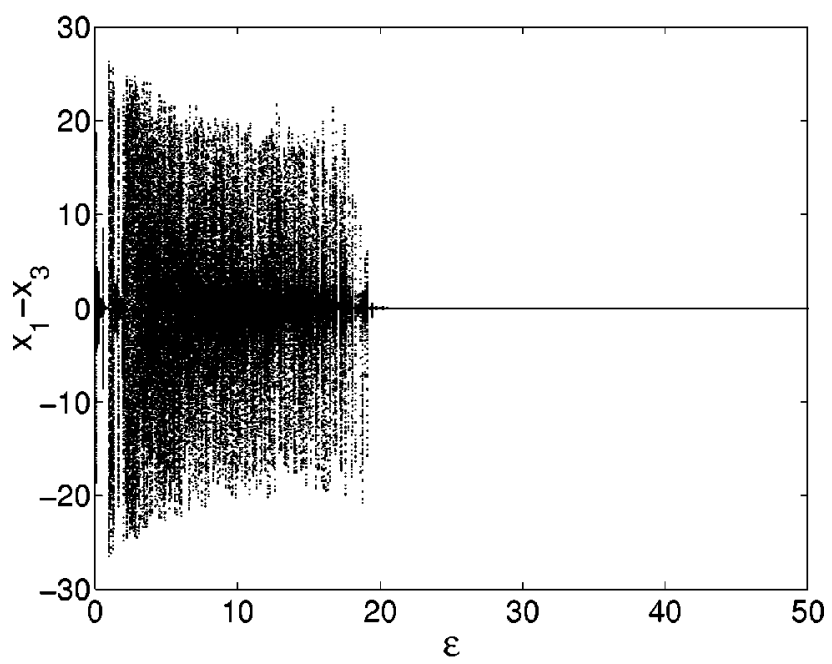

(b)

FIG. 6. Three Lorenz-like systems. (a) Bifurcation diagram for the dependence of $x_{1}-x_{2}$ on changing $\varepsilon$. There is no synchronization regime for any $\varepsilon$. (b) Bifurcation diagram for $x_{1}-x_{3}$ on changing $\varepsilon$. For $\varepsilon \approx 19$, partial synchronization appears, and is preserved for infinite $\varepsilon$.

$$
\begin{gathered}
\dot{y}_{2}=x_{2}+a y_{2}, \\
\dot{z}_{2}=b+\left(x_{2}-c\right) z_{2} .
\end{gathered}
$$

As in example $\mathrm{A}$, the functions $L\left(x_{1}\right)$ and $L\left(x_{2}\right)$ have a singularity at the points $x_{1,2}=c$, and therefore two equilibrium points $E_{1}$ and $E_{2}$ exist out of the diagonal and are preserved for any $\varepsilon$.

A bifurcation diagram for the dependence of $x_{1}-x_{2}$ on the coupling parameter $\varepsilon$ is shown in Fig. 7(a). For $\varepsilon$ $\approx 0.16$ the diagonal $M_{2,1}$ becomes locally stable and attracts all trajectories, except the stable manifolds of the two saddle foci $E_{1}$ and $E_{2}$. Under further increase of $\varepsilon$ this synchronized regime is preserved up to $\varepsilon \approx 1.9$, but for $\varepsilon>1.9$ the diagonal loses its local stability and an unsynchronized regime appears. 


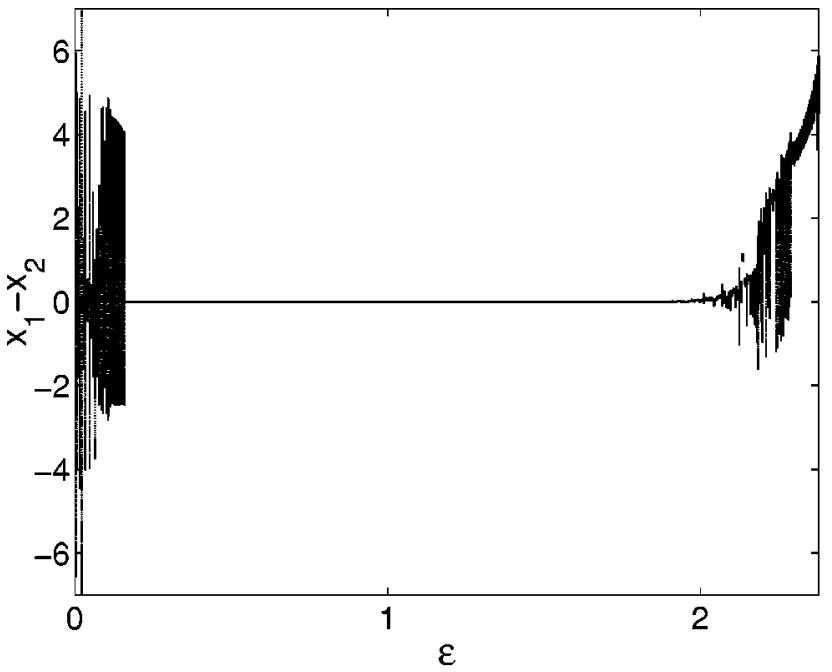

(a)

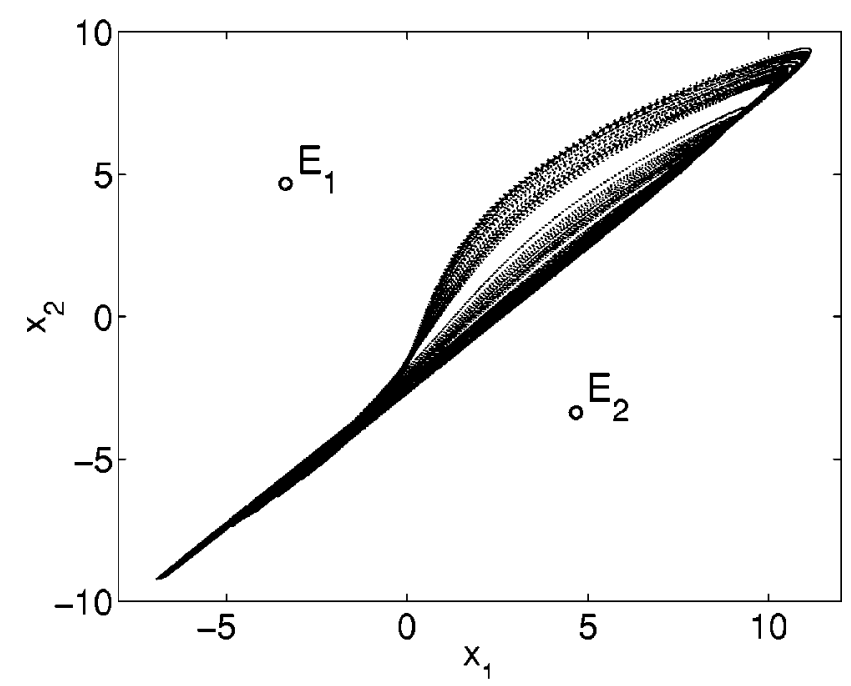

(b)

FIG. 7. Two Rössler systems. (a) Bifurcation diagram for the dependence of $x_{1}-x_{2}$ on $\varepsilon$. (b) Projection of the nonsynchronized trajectory on the plane $x_{1}, x_{2}$. Saddle foci with one-dimensional unstable manifolds, $E_{1}$ and $E_{2}$ are symmetrical with respect to the diagonal.

As in the above mentioned figures, the difference between corresponding variables ( $x_{1}$ and $x_{2}$, for example) of the subsystems is plotted vertically for each fixed value of $\varepsilon$ for fixed initial conditions $\left(x_{1} \neq x_{2}\right)$ at each step of $\varepsilon$. Figure 7(b) presents a projection of a nonsynchronized chaotic trajectory of Eq. (6.1) on the plane $\left(x_{1}, x_{2}\right)$ for parameters $a$ $=0.2, b=0.2, c=5.7$, and $\varepsilon=2.2$.

\section{B. Three coupled Rössler systems}

Now we consider three diffusively coupled Rössler systems

$$
\begin{gathered}
\dot{x}_{i}=-\left(y_{i}+z_{i}\right)+\varepsilon\left(x_{i+1}-2 x_{i}+x_{i-1}\right), \\
\dot{y}_{i}=x_{i}+a y_{i},
\end{gathered}
$$

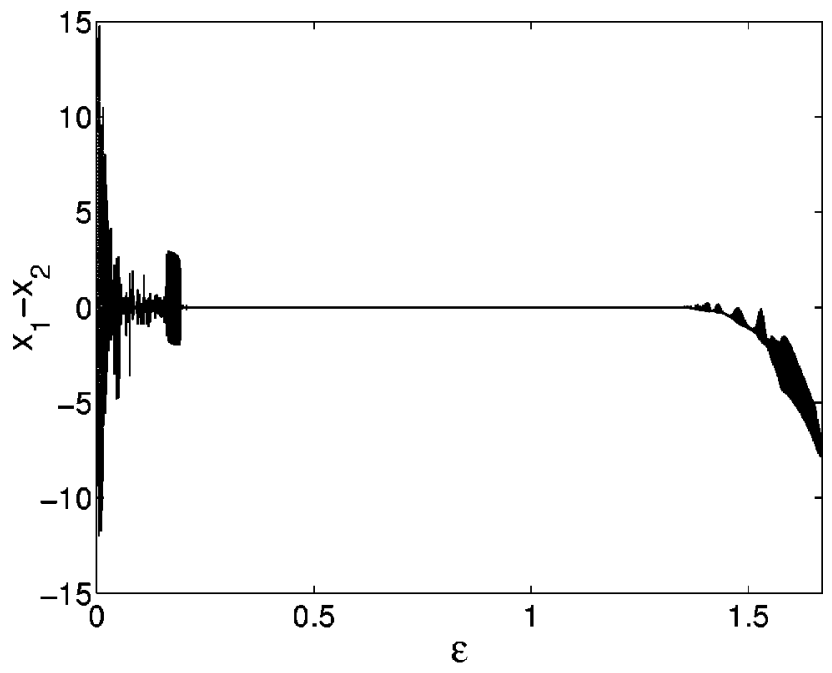

(a)

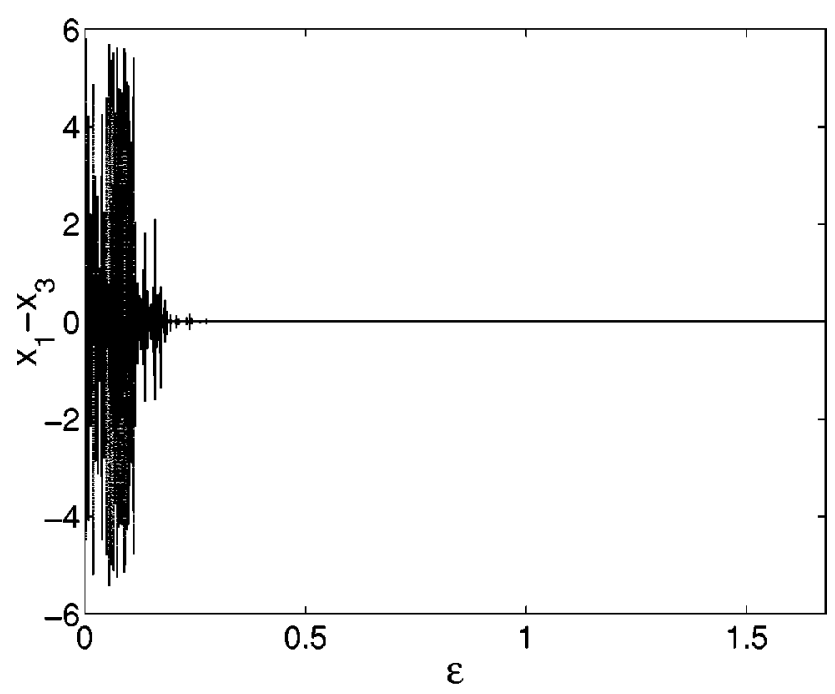

(b)

FIG. 8. Three Rössler systems. (a) Bifurcation diagram for the dependence of $x_{1}-x_{2}$ on $\varepsilon$. (b) Bifurcation diagram for the dependence of $x_{1}-x_{3}$ on changing $\varepsilon$. For $\varepsilon>1.38$, partial synchronization occurs. The other parameters are $a=0.2, b=0.2$, and $c=4.7$.

$$
\dot{z}_{i}=b+\left(x_{i}-c\right) z_{i}, \quad i=1,2,3
$$

with zero-flux BC's $x_{0}=x_{1}$ and $x_{4}=x_{3}$.

Similarly to example A, and in accordance with theorem 6, the individual subsystems of Eqs. (6.2) cannot be globally synchronized.

As in the case of the two coupled Rössler systems, with increasing $\varepsilon$ the diagonal $M_{3,1}$ becomes locally stable (for $\varepsilon \approx 0.2$ ), and attracts all trajectories besides the stable manifolds of the two saddle foci $E_{1}$ and $E_{2}$. Becoming stable, the invariant manifold $M_{3,2}$ contains the diagonal $M_{3,1}$ and the equilibria. All trajectories of system (6.2) in a neighborhood of $M_{3,1}$ in $M_{3,2}$ reach the diagonal $M_{3,1} . M_{3,1}$ remains stable up to $\varepsilon \approx 1.38$. For $\varepsilon>1.38$ it loses its stability [as shown in Fig. 8(a)], but $M_{3,2}$ remains stable [see Fig. 8(b)], and in this case we have the phenomenon of partial synchronization. Figure 9 shows the stable invariant manifold $M_{3,2}=\left\{x_{1}\right.$ 


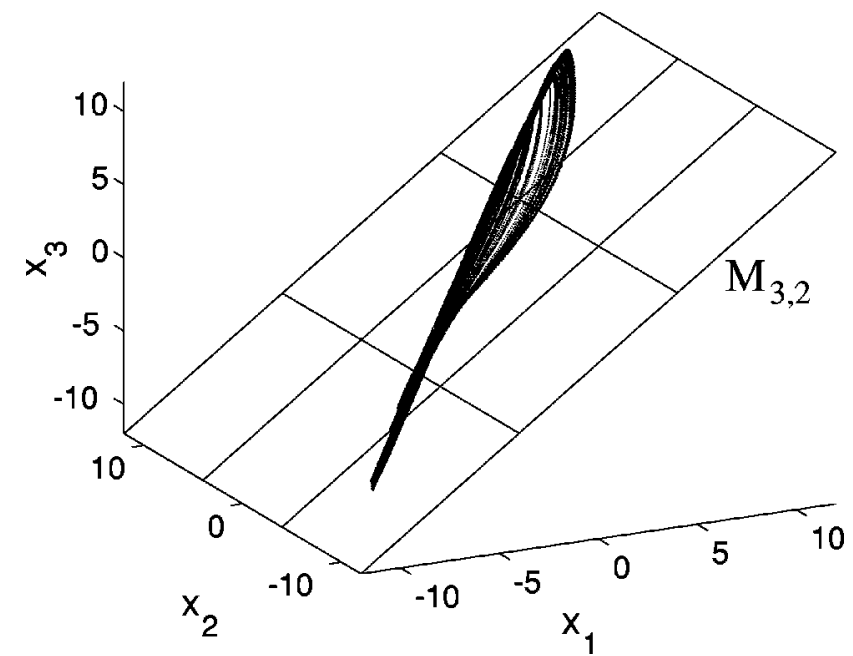

FIG. 9. (a) Projection of a chaotic trajectory into the stable invariant manifold $M_{3,2}\left[M_{3,1}\right.$ lost its stability with increasing coupling $(\varepsilon=1.4)]$.

$\left.=x_{3}, y_{1}=y_{3}, z_{1}=z_{3}\right\}$ which attracts all trajectories of the system (6.2). This invariant manifold corresponds to the synchronization between the first and third oscillators. Lying in it, a chaotic attractor defines the nonsynchronized regime between the variables of the first (the third) and second elements.

\section{Seven coupled Rössler systems}

Now we consider the case of a prime number $N$ of diffusively $x$-coupled Rössler systems and zero-flux BC's. For definiteness we chose $N=7$.

Due to theorem 1 and to the embedding equation (2.7), we have the same phenomenon as for the three coupled systems. Numerical simulation shows that with increasing coupling, the full local synchronization regimes loses its stability due to desynchronization bifurcations. Containing the unstable diagonal and remaining globally stable (for some range of coupling parameter $\varepsilon$ ), the invariant manifold $M_{7,4}=\left\{U_{1}\right.$ $\left.=U_{7}, U_{2}=U_{6}, U_{3}=U_{5}\right\}$ determines the partial synchronization of dimension 4.

Figure 10 presents the established cluster synchronization regime in the chain. This spatiotemporal pattern, with chaotic time dependent amplitudes of the individual oscillators, defines four clusters and synchronization in three pairs of elements. The middle (fourth) element is nonsynchronized, and defines a separate cluster.

\section{GENERALIZATION}

Let us conclude the present investigation by mentioning that our results admit the following generalizations.

(1) Theorems 1-3 and all embeddings [Eqs. (2.7)-(2.12)] are valid for system (1.1), where the time derivative stands for any linear differential or difference operator $D$. In particular, when $d u_{i} / d t$ denotes the difference $\left(\bar{U}_{i}-U_{i}\right)$, where $\bar{U}_{i}$ is the next iterate of $U_{i}$, system (1.1) becomes an array of locally coupled maps.

(2) Theorems 1-3 and the embeddings are valid for the system

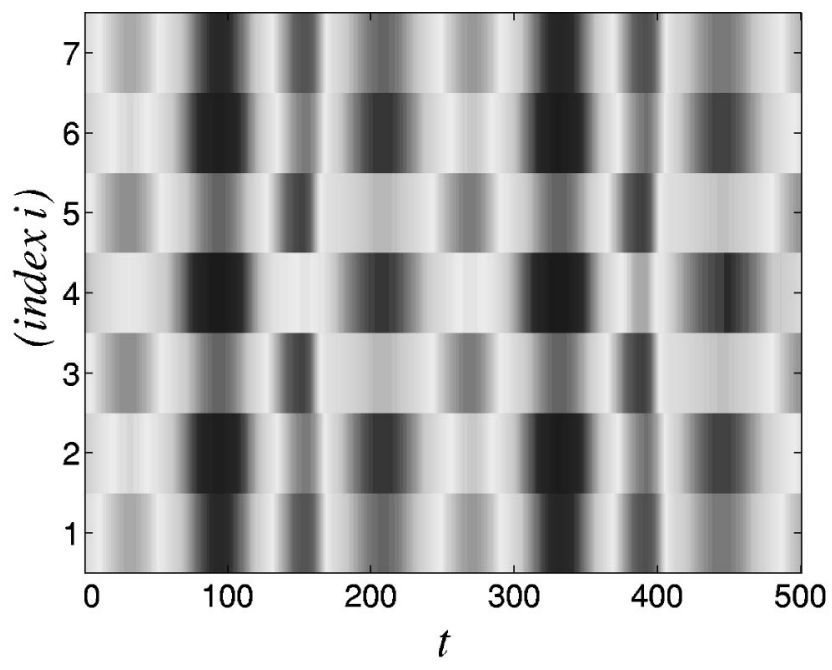

FIG. 10. Established cluster synchronization in the chain of seven diffusively coupled Rössler systems. Oscillators of the array are synchronized in pairs symmetrically around the middle (fourth) element, which remains nonsynchronized and defines one separate cluster. The parameters are $a=0.2, b=0.2, c=5.7$, and $\varepsilon=1.16$. Different shades of gray represent different ranges of amplitudes of $x_{i}(t)$.

$$
D U_{i}=G\left(U_{i}\right)+C\left(U_{i-1}, U_{i}, U_{i+1}\right), \quad i=1,2, \ldots, N
$$

with zero-flux BC's, where $U, G$, and $C$ are $m$ vectors, and the arbitrary function $C(U, V, W)$ satisfies the conditions of symmetry

$$
C(U, U, U)=0, \quad C(U, V, W)=C(W, V, U) .
$$

For example, function $C$ may be written both in the form of a linear coupling (vector diffusive coupling [10])

$$
C=S\left(U_{i-1}-2 U_{i}+U_{i+1}\right),
$$

and in the form of a nonlinear Kaneko-type coupling [19]

$$
C=S\left(G\left(U_{i-1}\right)-2 G\left(U_{i}\right)+G\left(U_{i+1}\right)\right),
$$

where the $m \times m$ matrix $S$ picks out the combinations of coupled vector coordinates.

(3) The symmetries of synchronized oscillations are also valid for the cases of plane and volume lattices of diffusively coupled continuous (or discrete) time dynamical systems. That is, in the case of a two-dimensional lattice, horizontal and vertical lines of the lattice play the roles of separate oscillators forming the clusters defined by theorems $1-3$. In the case of a three-dimensional lattice, the separate oscillators involved in the cluster synchronization regime are introduced by two-dimensional lattices in three volume directions. Obviously, all the above mentioned cases of coupled systems are subjects for future study.

\section{CONCLUSIONS}

We have discovered the family of embedded invariant manifolds of an array of diffusively coupled identical dynamical systems, and discussed the question of global stabil- 
ity of synchronization manifolds. Obviously these embedded manifolds come from the symmetry of both coupling and boundary conditions, independently of the dynamics of the individual element. In phase space they make up a "skeleton" defining a strict set of possible modes of cluster synchronization. Realization of these modes depends on the vector field of the single system, on whether the concrete forms of both systems in a manifold are self-similar or not, and on the corresponding variational stability equation.

The most interesting feature of the embeddings is the essential dependence on the number of oscillators $N$. For $N$ $=p^{k}$, where $p$ is a prime number $(2,3,5, \ldots)$, the straight self-similar embedding with the ordering of dimensions $p^{k}$ $\rightarrow p^{k-1} \rightarrow \cdots \rightarrow p$, as well as asymmetrical terminal enclosure $p \rightarrow(p+1) / 2 \rightarrow 1$, allow us to make the main conclusion related to the cluster manifolds embeddings: system (1.1) with zero-flux boundary conditions and prime numbers of elements plays the role of the basic unit in cluster synchronization. We emphasize that if $p$ is a cofactor of $N$, then system (1.1), with $N$ oscillators, may be considered as a selfsimilar extension of itself with $p$ elements.

We remark that the problem of manifold stability is intimately related to the problem of synchronization persistence under a parameter mismatch between the oscillators $[2,11-$ 13]. That is, if a manifold $M_{N, d}$ is strongly asymptotically stable (all Lyapunov exponents are bounded away to the left from zero) then this manifold is preserved under a small perturbation of system (1.1), and then the perturbed quasilinear manifold $\widetilde{M}_{N, d}$ [such that the distance $\left(\widetilde{M}_{N, d}, M_{N, d}\right)$ is small] defines the persistence of the cluster synchronization regime of coupled oscillators in the presence of a small parameter mismatch.

Finally we present some comments on the problem of the relation between equilibria and invariant manifolds. In one extreme case when system (1.1) is gradientlike, it has no bifurcations besides saddle-node bifurcations of equilibria. Hence for this system the bifurcation of global stability onset of each invariant manifold coincides with the bifurcation of some equilibria.

Conversely, in another extreme case a globally stable manifold $M_{N, d}$ may lose its local Lyapunov stability via bub- bling [32], when a transversal Lyapunov exponent of a chaotic trajectory lying in the manifold $M_{N, d}$ becomes positive. Then the global stability onset of this manifold may be far from the bifurcational set of equilibria. In general, we conjecture that the solution of the problem of the relation between equilibria bifurcations and conditions of global stability of invariant manifolds lies between these extremes.

Another peculiarity appears when system (1.1) is considered as a discrete simplified model of spatially extended reaction-diffusion system defined by partial differential equations

$$
\begin{gathered}
\frac{\partial x}{\partial t}=P(x, y)+\frac{\partial^{2} x}{\partial s^{2}}, \quad \frac{\partial y}{\partial t}=Q(x, y), \\
\left.\frac{\partial U}{\partial s}\right|_{s=0}=0,\left.\quad \frac{\partial U}{\partial s}\right|_{s=a}=0
\end{gathered}
$$

on the interval $[0, a]$ of spatial variable $s$. The mode of full synchronization of the extended system (8.1) becomes a homogeneous solution, and equilibria of system (1.1), being the trajectories of Eq. (3.9), become the solutions of the boundary-value problem for Eqs. (8.1) at $\partial U / \partial t \equiv 0$.

The mode of partial synchronization defined by a symmetrical manifold of Eq. (1.1) in the case of the system (8.1) is related to similar symmetrical solutions satisfying conditions $x(s, t)=x(a-s, t)$. The important case, where system (1.1) is self-similar, has an analog for the system (8.1) as symmetrical solutions at intervals of some wavelength. For example, for $N=2^{k}$ these lengths are $a / 2^{j}, j=1,2, \ldots, k$. Note that the problem of stability of such solutions of Eqs. (8.1) is harder than for system (1.1).

\section{ACKNOWLEDGMENTS}

I.B. acknowledges the hospitality of the Department of Electrical Engineering of the Swiss Federal Institute of Technology (EPFL), and the financial support of the Swiss government. This work was also supported in part by RFFI (Grant No. 99-01-01126) and by the grant "Universities of Russia"' (No. 1905).
[1] H. Fujisaka and T. Yamada, Prog. Theor. Phys. 69, 32 (1983); 72, 885 (1984).

[2] V. S. Afraimovich, N. N. Verichev, and M. I. Rabinovich, Izv. Vyssh. Uchebn. Zaved. Radiofiz. 29, 795 (1986).

[3] L. M. Pecora and T. L. Carroll, Phys. Rev. Lett. 64, 821 (1990).

[4] J. F. Heagy, T. L. Carroll, and L. M. Pecora, Phys. Rev. E 50, 1874 (1994).

[5] T. L. Carroll and L. M. Pecora, Physica D 67, 126 (1993).

[6] V. N. Belykh, N. N. Verichev, L. J. Kocarev, and L. O. Chua, J. Circuits Syst. Comput. 3, 579 (1993).

[7] J. F. Heagy, L. M. Pecora, and T. L. Carroll, Phys. Rev. Lett. 74, 4185 (1995)

[8] A. Sherman, Bull. Math. Biol. 56, 811 (1994).

[9] L. M. Pecora, T. L. Carroll, G. A. Johnson, D. J. Mar, and J. F. Heagy, Chaos 7, 520 (1997).
[10] L. M. Pecora, Phys. Rev. E 58, 347 (1998).

[11] G. A. Johnson, D. J. Mar, T. L. Carroll, and L. M. Pecora, Phys. Rev. Lett. 80, 3956 (1998).

[12] N. F. Rulkov, M. M. Sushchik, L. S. Tsimring, and H. D. I. Abarbanel, Phys. Rev. E 51, 980 (1995).

[13] H. D. Abarbanel, N. F. Rulkov, and M. M. Sushchik, Phys. Rev. E 53, 4528 (1996).

[14] V. N. Belykh and E. Mosekilde, Phys. Rev. E 54, 3196 (1996).

[15] N. F. Rulkov, Chaos 6, 262 (1996).

[16] V. N. Belykh, I. V. Belykh, and N. N. Verichev, Izv. Vyssh. Uchebn. Zaved. Radiofiz. 7, 912 (1997).

[17] A. L. Fradkov and A. Y. Markov, IEEE Trans. Circuits Syst., I: Fundam. Theory Appl. 44, 905 (1997).

[18] A. S. Dmitriev, M. Shirokov, and S. O. Starkov, IEEE Trans. Circuits Syst., I: Fundam. Theory Appl. 44, 918 (1997).

[19] K. Kaneko, Physica D 41, 137 (1990); 54, 5 (1991); 55, 368 (1992); 75, 55 (1994). 
[20] K. Pyragas, Phys. Rev. E 54, R4508 (1996).

[21] M. S. Vieira and A. J. Lichtenberg, Phys. Rev. E 56, R3741 (1997)

[22] D. H. Zanette and A. S. Mikhailov, Phys. Rev. E 57, 276 (1998).

[23] S. C. Manrubia and A. S. Mikhailov, Phys. Rev. E 60, 1579 (1999).

[24] M. Hasler, Yu. Maistrenko, and O. Popovych, Phys. Rev. E 58, 6843 (1998).

[25] Z. Liu, S. Chen, and B. Hu, Phys. Rev. E 59, 2817 (1999).

[26] Chua's Circuit: a Paradigm for Chaos, edited by R. Madan (World Scientific, Singapore, 1993).

[27] M. Hasler, Philos. Trans. R. Soc. London, Ser. A 353, 115 (1995).

[28] V. S. Anishenko, T. E. Vadivasova, D. E. Postnov, and M. A. Safonova, Int. J. Bifurcation Chaos Appl. Sci. Eng. 2, 633 (1992).
[29] M. G. Rosenblum, A. S. Pikovsky, and J. Kurths, Phys. Rev. Lett. 76, 1804 (1996).

[30] M. G. Rosenblum, A. S. Pikovsky, and J. Kurths, Phys. Rev. Lett. 78, 4193 (1997).

[31] J. C. Alexander, J. A. Yorke, Z. You, and I. Kan, Int. J. Bifurcation Chaos Appl. Sci. Eng. 2, 795 (1992); J. C. Alexander, B. R. Hunt, I. Kan, and J. A. Yorke, Ergod. Th. Dyn. Syst. 16, 651 (1996); Yu. L. Maistrenko, V. L. Maistrenko, A. Popovich, and E. Mosekilde, Phys. Rev. Lett. 80, 1638 (1998).

[32] P. Ashwin, J. Buescu, and I. Stewart, Phys. Lett. A 193, 126 (1994); Nonlinearity 9, 703 (1996); J. F. Heagy, T. L. Carroll, and L. M. Pecora, Phys. Rev. E 52, R1253 (1995); M. Hasler and Yu. Maistrenko, IEEE Trans. Circuits Syst., I: Fundam. Theory Appl. 44, 856 (1997).

[33] L. Yu, E. Ott, and Q. Chen, Physica D 53, 102 (1992); N. Platt, E. A. Spiegel, and C. Tresser, Phys. Rev. Lett. 70, 279 (1993).

[34] V. N. Belykh, Sbornik: Mathematics 186, 311 (1995). 